\title{
Low-frequency radio monitoring of microquasars ${ }^{\star}$
}

\author{
M. Pandey ${ }^{1,2}$, A. P. Rao ${ }^{3}$, C. H. Ishwara-Chandra ${ }^{3}$, P. Durouchoux ${ }^{4}$, and R. K. Manchanda ${ }^{5}$ \\ 1 CNRS FRE 2591/CEA Saclay, DSM/DAPNIA/SAP, 91191 Gif-sur-Yvette Cedex, France \\ e-mail: mamtapan@gmail.com \\ 2 Dept. of Physics, Mumbai University, Mumbai - 400 098, India \\ 3 NCRA, TIFR, Post Bag 3, Ganeshkhind, Pune - 411 007, India \\ 4 ESIEA-DRDI, 11 rue Baudin, 92400 Ivry-sur-Seine, France \\ 5 Tata Institute of Fundamental Research, Mumbai - 400005, India
}

Received 19 June 2006 / Accepted 9 October 2006

\section{ABSTRACT}

\begin{abstract}
Microquasars are radio-emitting X-ray binaries (REXBs) with a radio morphology like quasars and high X-ray luminosity. Sixteen known microquasar candidates were extensively monitored for the first time at low radio frequencies using the Giant Meter-wave Radio Telescope (GMRT) between 6-June 2003 and 22-Jan. 2005 at 0.235/0.61 (simultaneous) and 1.28 GHz. Nine out of sixteen sources were detected positively by the GMRT including all six high-mass X-ray binaries (HMXBs) and three low-mass X-ray binaries (LMXBs). Among the nine sources emitting at low frequencies, six are persistent in radio and three are transient at radio wavelengths. In the case of four persistent radio sources (Scorpius X-1, Cyg X-1, Cyg X-3, and LSI+61303) the contemporaneous data suggests a spectral turnover $\left(S_{v}=v^{\alpha}, \alpha>0\right)$ and agrees with the synchrotron self absorption (SSA) effect expected at lower frequencies. The radio spectra of SS433 and LS5039 show a power law decay $\left(S_{v}=v^{\alpha}, \alpha<0\right)$ with no signature of SSA even at the very low frequency of $0.235 \mathrm{GHz}$. This unique result suggests either that these sources are scatter-broadened at lower frequencies or that the low-frequency radio emission from these sources are superimposed by the emission from an extended region located near these sources. Five sources, GRO J1655-40, XTE J1118+480, 1E1740.7-2942, XTE J1748-288, and GRS 1758-258 were never detected during our observations, thus suggesting that they show the SSA effect at lower frequencies or that they are too faint to be detected at GMRT frequencies. Because interstellar scintillation becomes dominant at low frequencies and may lead to flux-density fluctuations, the scintillation time scale for each microquasar was calculated and compared to the variability time scale in the data. We confirm from these studies that Cyg X-1 and SS433 are most likely affected by scintillation and that LSI+61303, LS 5039, Sco X-1, and XTE J1118+480 may possibly be affected by scintillation. A comparative study of the radio luminosity from centimeter-(GHz) to meter-wavelength $(\mathrm{MHz})$ suggests a decrease by a few orders of magnitude as one goes lower in frequency. We have also plotted the RXTE/ASM X-ray light curve for all the sixteen known microquasars. Based on the ASM data, the X-ray light curve can be classified as: (a) persistent, (b) quasi-persistent or (c) transient. From the analysis of these types and the information about their companion star, the persistent or transient nature of the radio jet can be confirmed. This paper provides a general review of the main observational results obtained up to now, as well as different models for the production of low-frequency radio emissions from these sources.
\end{abstract}

Key words. radiation mechanisms: non-thermal - acceleration of particles - instrumentation: interometers methods: observational - binaries: general - ISM : jets and outflows

\section{Introduction}

$\mathrm{X}$-ray binaries are very luminous binary stars were the high energy emissions are produced by matter falling from a normal star to a compact star, which is a neutron star (NS) or a black hole $(\mathrm{BH})$. The in-falling matter releases gravitational potential energy up to several tens of per cent of its rest mass as $\mathrm{X}$-rays. The soft thermal component of BH X-ray spectra originates in an optically thick, geometrically thin accretion disc (Shakura \& Sunyaev 1973), whereas the power law component is produced by Comptonization of "seed" photons in the hot, rarefied "corona" of (quasi-)thermal electrons (Shapiro et al. 1976; Sunyaev \& Titarchuk 1980; Haardt \& Maraschi 1991; Poutanen \& Svensson 1996). These sources exist in various X-ray states preliminarie classified into low/hard, intermediate, and high/soft state. The matter is occasionally ejected from these systems due to non-thermal processes giving rise to radio emissions. The radio emissions are known to be enhanced in the low/hard state

* Table 2 is only available in electronic form at http://www . aanda. org and quenched in the high/soft state. The existence of corona at the base of the jet has been well-established from the evidence for a turnover in the infrared - optical band, where the flat-toabsorbed $(\alpha>0)$ radio spectrum seems to be connected to an optically thin component extending up to X-rays (Corbel \& Fender 2002; Markoff et al. 2003a,b, and references therein).

The X-ray binaries are further subdivided into HMXBs and LMXBs based upon the mass of their companion star. HMXBs have a massive secondary star, mainly super-giants, with vigorous stellar winds. In these systems, a primary star of a few solar masses generally accretes the matter from the stellar winds of the huge companion stars. The production of X-rays can be induced even without mass transfer through the Roche lobe. This requires the involvement of strong magnetic fields. At radio wavelengths, HMXBs are mostly persistent emitters. In the case of LMXBs, the companions are low mass secondary stars, mainly sub-giants. The X-rays are characterized by the production of bursts rather than pulses, which in turn implies the involvement of weak magnetic fields. LMXBs show transient behavior at radio wavelengths. 

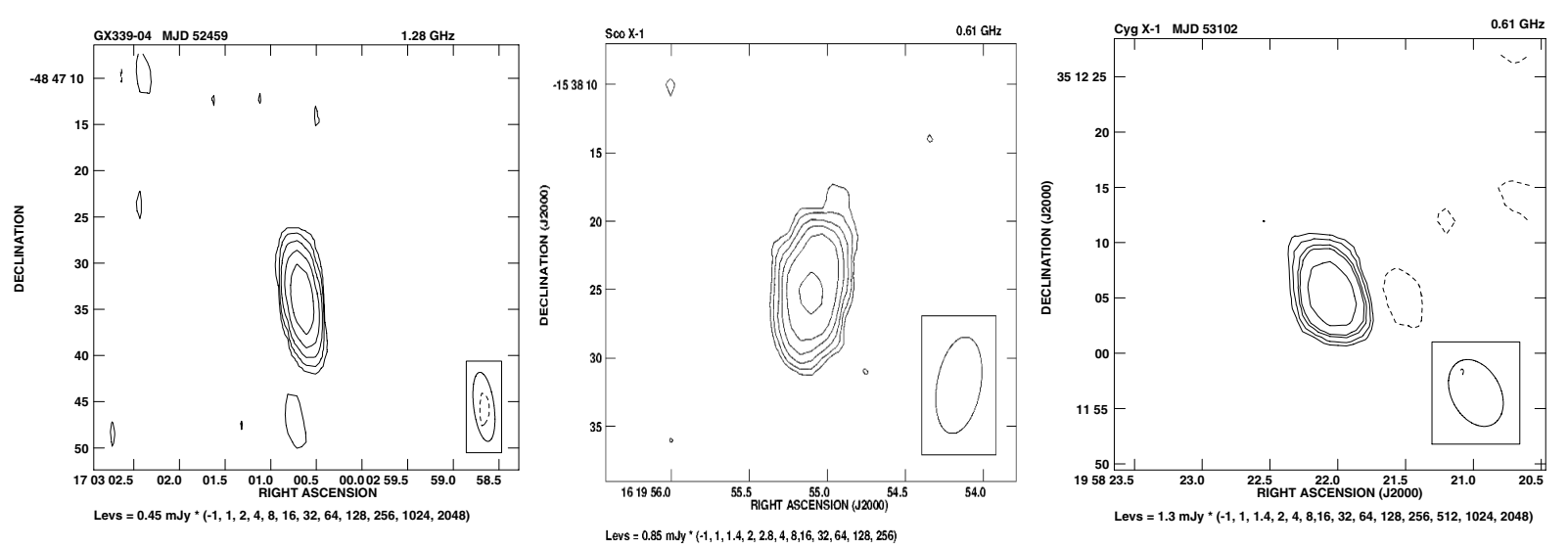

Fig. 1. Radio image of microquasars GX339-04 (left), Sco X-1 (middle), and Cyg X-1 (right) observed with GMRT.

Among the above-mentioned classes, a group of $\sim 43$ X-ray binaries emitting at radio wavelengths are known as REXBs (Paredes et al. 2005). Microquasars belong to this category. They have characteristics in common with quasars, variable radio emission often seen as radio jets and an accretion disc surrounding a $\mathrm{BH}$. The spectral analysis of these sources suggests that the radio emission originates during a synchrotron process (Hjellming et al. 1988; Han et al. 1995). Radio jets have been commonly observed in these systems that apparently show superluminal motions during the flaring stages, e.g. GRS 1915+105 (Mirabel et al. 1994). They show rapid day-to-day variable radio flux density during the flaring phase; however, these sources are expected to have a nearly constant flux in the quiescent phase. It is important to investigate the basic behavior of the X-ray light curve and the radio spectra from centimeter to meter-wavelengths in these sources. Various systematic studies of micro-quasars have been carried at centimeter wavelengths ( $\geq 2 \mathrm{GHz}$ ) (Fender et al. 2000); however, the meter-wavelength characteristics not yet have explored.

We carried out a systematic low-frequency monitoring of microquasars using the GMRT (Pandey et al. 2004) for the first time, to explore the low-frequency behavior of these jet sources. We investigated in detail the radio spectra of the persistent radio sources in order to look for the correlation between meter - and the available near-simultaneous radio data at centimeter wavelength. Significant variability in the flux density at low radio frequencies $(v \leq 1.28 \mathrm{GHz})$ was seen for these sources during our observations. Using the RXTE/ASM data, we show that the X-ray light curve of all sixteen known microquasars can be classified in three categories. From the analysis of these types and their companion stars, the nature of the radio emission can be inferred for these sources. We present the results of our analysis of both the radio and X-ray data on HMXBs and LMXBs in Sect. 2. In Sect. 3 we discuss the scintillation effects on the low-frequency radio data and compare the radio properties at high and low radio frequencies. A detailed discussion of the radio spectra of persistent microquasars and the models for radio emission from these sources are presented in detail in Sect. 4.

\section{Observations and analysis}

\subsection{Radio observations}

The GMRT consists of thirty radio antennas distributed over a region of about $25 \mathrm{~km}$, with fourteen of the antennas located within a diameter of about one $\mathrm{km}$ and the rest arranged in $3 \mathrm{arms}$ each of $14 \mathrm{~km}$ length, shaped as an irregular Y. This arrangement provides the necessary $u v$ coverage for mapping both compact and extended sources. The ratio of longest to the shortest baseline is $\sim 500$ with the shortest baseline $\sim 50 \mathrm{~m}$. The radio observations were carried out simultaneously at $0.235 / 0.61 \mathrm{GHz}$ and also at $1.28 \mathrm{GHz}$ with a bandwidth of $6 / 16$ and $32 \mathrm{MHz}$, respectively. The flux density scale was set by observing the primary calibrators 3C 286 and 3C 48. Phase calibrators were integrated with 25 min scans on each microquasar. The integration time was $16 \mathrm{~s}$. The visibility data recorded from GMRT was converted into FITS files and analyzed using the Astronomical Image Processing System (AIPS). Bad data due to antennabiased or baseline-biased problems were flagged using UVFLG. The data were then calibrated and images of the fields formed by Fourier inversion and CLEANing (IMAGR). The visibility data were then used to generate the continuum maps (Fig. 1).

As seen in Fig. 1 the microquasars observed with GMRT are unresolved point sources. All the maps presented in this paper have been corrected for the system temperature corrections at low frequencies. A self calibration of the data was performed to correct for phase-related errors and to improve the image quality. Tables 1-3 summarizes details of our observations of transient radio sources with no detections, transient radio sources with rare detections, and persistent radio sources, respectively, using the GMRT simultaneously at $0.235 / 0.61 \mathrm{GHz}$ and at $1.28 \mathrm{GHz}$ between June 2003 and Feb. 2005. Flux densities of all the known microquasars observed with the GMRT are listed in the tables except for GRS 1915+105. A detailed study of this source will be presented in Pandey et al. (2006a) as it is beyond the scope of this paper. In Table 4, Cols. 1-3 list the general properties of micro-quasars. Cols. 4 and 5 show the radio behavior of these sources at centimeter and meter-wavelengths, respectively.

To supplement the data from GMRT, we also used the available radio data on these sources with various telescopes observing at high frequencies. The Ryle telescope is continuously monitoring a few microquasars at $15 \mathrm{GHz}$ on a daily basis. The typical uncertainty is $2 \mathrm{mJy}+3 \%$ in the flux scaling. Nearly simultaneous data on a few microquasars is used in this paper. The Green Bank Interferometer (GBI), West Virginia, operated by NRAO, provides data for a number of X-ray sources that were monitored during its several years of operation between 1996 and 2000. The radio light curve at $2.2 \mathrm{GHz}$ and $8 \mathrm{GHz}$ was obtained with $10 \mathrm{~min}$ averaging time on each source. The typical rms is $20 \mathrm{mJy}$ at $2.2 \mathrm{GHz}$ and $15 \mathrm{mJy}$ at $8 \mathrm{GHz}$. The mean radio flux for the persistent radio sources was obtained from the 
Table 1. Details of observations on transient radio sources XTE J1118+480, GRO J1655-40, XTE J1748-288, GRS 1758-258, and 1E1740.7-2942 with the GMRT.

\begin{tabular}{|c|c|c|c|c|c|}
\hline Source & MJD & $\begin{array}{l}\text { Freq. } \\
\text { (MHz) }\end{array}$ & $\overline{\text { Detection }}$ & $\begin{array}{l}S_{v} \\
(\mathrm{mJy})\end{array}$ & $\begin{array}{l}\text { rms } \\
\left(\mathrm{mJy} \mathrm{b}^{-1}\right)\end{array}$ \\
\hline \multirow[t]{9}{*}{ XTE J1118+480 } & 52459 & 1280 & No & $<0.33$ & 0.11 \\
\hline & 52694 & $610 / 235$ & No & $<2.55 /<5$ & $0.85 / 1.6$ \\
\hline & 52699 & $610 / 235$ & No & $<2.25 /<4.77$ & $0.75 / 1.59$ \\
\hline & 52702 & $610 / 235$ & No & $<1.95 /<6.60$ & $0.65 / 2.20$ \\
\hline & 52855 & 1280 & No & $<0.54$ & 0.18 \\
\hline & 52898 & $610 / 235$ & No & $<3.54 /<6.99$ & $1.18 / 2.33$ \\
\hline & 53220 & $610 / 235$ & No & $<3.78 /<6.06$ & $1.26 / 2.02$ \\
\hline & 53377 & $610 / 235$ & No & $<3.51 /<9.12$ & $1.17 / 3.04$ \\
\hline & 53391 & $610 / 235$ & No & $<3.99 /<8.34$ & $1.33 / 2.78$ \\
\hline \multirow[t]{7}{*}{ GRO J1655-40 } & 52459 & 1280 & No & $<0.36$ & 0.12 \\
\hline & 52694 & $610 / 235$ & No & $<2.04 / 6.03$ & $0.68 / 2.01$ \\
\hline & 52699 & $610 / 235$ & No & $<1.68 /<5.67$ & $0.56 / 1.89$ \\
\hline & 52702 & $610 / 235$ & No & $<2.25 /<6.60$ & $0.75 / 2.20$ \\
\hline & 52849 & 1280 & No & $<0.27$ & 0.09 \\
\hline & 52855 & 1280 & No & $<0.30$ & 0.10 \\
\hline & 53377 & $610 / 235$ & No & $<2.22 /<6.9$ & $0.74 / 2.3$ \\
\hline \multirow{5}{*}{ XTE J1748-288 } & 52849 & 1280 & No & $<0.237$ & 0.079 \\
\hline & 53127 & $610 / 235$ & No & $<3.18 /<6.39$ & $1.06 / 2.13$ \\
\hline & 53107 & $610 / 235$ & No & $<3.03 /<3.78$ & $1.01 / 1.26$ \\
\hline & 53379 & $610 / 235$ & No & $<2.94 /<6.09$ & $0.98 / 2.03$ \\
\hline & 53391 & $610 / 235$ & No & $<3.06 /<6.27$ & $1.02 / 2.09$ \\
\hline \multirow[t]{11}{*}{ GRS 1758-258 } & 52459 & 1280 & No & $<0.66$ & 0.22 \\
\hline & 52694 & $610 / 235$ & No & $<3.21 /<4.38$ & $1.07 / 1.46$ \\
\hline & 52699 & $610 / 235$ & No & $<2.34 /<5.85$ & $0.78 / 1.95$ \\
\hline & 52702 & $610 / 235$ & No & $<2.40 /<3.69$ & $0.80 / 1.23$ \\
\hline & 52707 & $610 / 235$ & No & $<3.09 /<6.12$ & $1.03 / 2.04$ \\
\hline & 52849 & 1280 & No & $<0.60$ & 0.2 \\
\hline & 52855 & 1280 & No & $<0.27$ & 0.09 \\
\hline & 53127 & $610 / 235$ & No & $<3.48 /<4.35$ & $1.16 / 1.45$ \\
\hline & 53379 & $610 / 235$ & No & $<2.91 /<3.93$ & $0.97 / 1.31$ \\
\hline & 53391 & $610 / 235$ & No & $<3.42 /<6.27$ & $1.14 / 2.09$ \\
\hline & 53392 & $610 / 235$ & No & $<3.33 /<5.49$ & $1.11 / 1.83$ \\
\hline \multirow{11}{*}{ 1E1740.7-2942 } & 52459 & 1280 & No & $<0.27$ & 0.09 \\
\hline & 52694 & $610 / 235$ & No & $<3.27 /<4.62$ & $1.09 / 1.54$ \\
\hline & 52699 & $610 / 235$ & No & $<3.42 /<4.56$ & $1.14 / 1.52$ \\
\hline & 52702 & $610 / 235$ & No & $<3.66 /<6.18$ & $1.22 / 2.06$ \\
\hline & 52707 & $610 / 235$ & No & $<3.57 /<6.09$ & $1.19 / 2.03$ \\
\hline & 52849 & 1280 & No & $<0.39$ & 0.13 \\
\hline & 52855 & 1280 & No & $<0.6$ & 0.20 \\
\hline & 52897 & $610 / 235$ & No & $<3.63 /<6.12$ & $1.21 / 2.04$ \\
\hline & 53107 & $610 / 235$ & No & $<2.73 /<6.18$ & $0.91 / 2.06$ \\
\hline & 53379 & $610 / 235$ & No & $<3.12 /<4.17$ & $1.04 / 1.39$ \\
\hline & 53392 & $610 / 235$ & No & $<3.48 /<6.09$ & $1.16 / 2.03$ \\
\hline
\end{tabular}

three years of GMRT data at $0.235 / 0.61$ and $1.28 \mathrm{GHz}$ as listed in Tables 1-3 and four years of GBI radio data simultaneously at $2.2 \mathrm{GHz}$ and $8 \mathrm{GHz}$. The radio light curves from the GBI data at $2.2 \mathrm{GHz}$ and $8.2 \mathrm{GHz}$ for the persistent radio sources are plotted for reference in Figs. 2 and 3. It can be seen that LSI+61303, LS 5039, and Cyg X-1 show rare flaring events. A 26-days periodicity in the radio light curve of LSI+61303 is also seen, as reported in the literature. On the other hand, Sco X-1, SS433, and Cyg X-3 show frequent flaring events in the radio light curve.

The transient radio sources were not detected during most of our observations, hence no result is presented for these sources in this paper. However, a precise discussion of their general behavior is given in Sects. 2.2 and 3. The $3 \sigma$ upper limit is considered as the reference radio flux density for transient radio sources. It is important to note that a few transient radio sources (GRS 1915+105, V4641 Sgr, and GX339-04) were detected by GMRT during radio outbursts before 2003, and detailed results have been published elsewhere (Ishwara-Chandra et al. 2004;
Ishwara-Chandra et al. 2005a,b). In the case of V4641 Sgr and GX339-04, the sources were detected at radio wavelengths on a few occasions when they went in to low/hard X-ray states as shown in Fig. 4.

In Sect. 2.2 we discuss the classification of these sources based on the nature of their RXTE/ASM X-ray light curve, the mass of the companion star, and its direct connection to their nature at radio wavelengths.

\subsection{X-ray observations}

The RXTE All Sky Monitor provides the X-ray light curve for all the microquasars in three sub-energy bands (or "colors"), soft, medium, and hard. The X-ray light curve for each source was generated using the sum of intensities of all bands to look for a common pattern in these sources. Based on this study, we crudely grouped the light curves into 3 types: (A)- persistent X-ray light curve with no flaring events, (B)- quasi-persistent 
Table 3. Flux density of persistent radio sources Cyg X-1, Cyg X-3, Sco X-1, SS433, LS 5039, and LSI+61303 between Jun. $2003-J a n .2005$.

\begin{tabular}{|c|c|c|c|c|c|c|c|}
\hline Source & MJD & $\begin{array}{l}\text { Freq. } \\
(\mathrm{MHz})\end{array}$ & Detection & $\begin{array}{l}S_{v} \\
(\mathrm{mJy})\end{array}$ & $\alpha$ & $\begin{array}{l}S_{v(15 \mathrm{GHz})} \\
(\mathrm{mJy})\end{array}$ & $\begin{array}{l}\mathrm{rms} \\
\left(\mathrm{mJy} \mathrm{b}^{-1}\right)\end{array}$ \\
\hline \multirow{18}{*}{ Cyg X-1 } & 52796 & 1280 & No & $<0.93$ & & 13 & 0.31 \\
\hline & 52799 & 1280 & Yes & $10.1 \pm 0.96$ & & 22 & 0.3 \\
\hline & 52827 & $610 / 235$ & No & $<1.5 /<4.08$ & & 8 & $0.5 / 1.36$ \\
\hline & 52829 & $610 / 235$ & No & $<1.2 /<4.02$ & & 13 & $0.4 / 1.34$ \\
\hline & 52845 & 1280 & No & $<0.6$ & & 6 & 0.2 \\
\hline & 52848 & 1280 & No & $<0.9$ & & 6 & 0.3 \\
\hline & 52855 & 1280 & Yes & $5.40 \pm 0.21$ & & 22 & 0.2 \\
\hline & 52891 & $610 / 235$ & Yes & $5.80 \pm 1.63 / 7.36 \pm 2.02$ & -0.25 & 15 & $0.7 / 1.52$ \\
\hline & 53037 & 1280 & Yes & $9.20 \pm 0.04$ & & 15 & 0.2 \\
\hline & 53102 & $610 / 235$ & Yes & $9.07 \pm 0.43 / 10.01 \pm 2.43$ & -0.10 & 22 & $0.4 / 1.70$ \\
\hline & 53104 & $610 / 235$ & No & $<2.7 /<5.49$ & & 27 & $0.9 / 1.83$ \\
\hline & 53107 & $610 / 235$ & Yes & $10.03 \pm 0.12 / 7.81 \pm 1.83$ & 0.26 & 26 & $0.7 / 1.34$ \\
\hline & 53127 & $610 / 235$ & No & $<2.73 /<4.53$ & & 16 & $0.9 / 1.51$ \\
\hline & 53377 & $610 / 235$ & No & $<2.10 /<4.29$ & & 6 & $0.7 / 1.43$ \\
\hline & 53379 & $610 / 235$ & No & $<3.30 /<5.49$ & & 6 & $1.1 / 1.83$ \\
\hline & 53381 & $610 / 235$ & yes & $7.30 \pm 0.12 / 9.73 \pm 2.80$ & -0.30 & 16 & $0.7 / 1.62$ \\
\hline & 53391 & $610 / 235$ & yes & $8.40 \pm 0.12 / 11.97 \pm 1.66$ & -0.37 & 20 & $0.9 / 1.41$ \\
\hline & 53392 & $610 / 235$ & yes & $8.40 \pm 0.12 / 12.26 \pm 2.07$ & -0.39 & 16 & $0.8 / 1.55$ \\
\hline \multirow[t]{15}{*}{ Cyg X-3 } & 52797 & 1280 & Yes & $57.90 \pm 0.88$ & & 70 & 0.4 \\
\hline & 52800 & 1280 & Yes & $90.40 \pm 1.03$ & & 110 & 0.3 \\
\hline & 52830 & $610 / 235$ & Yes & $41.18 \pm 4.38 / 13.26 \pm 1.38$ & 1.19 & 115 & $0.8 / 1.24$ \\
\hline & 52849 & 1280 & Yes & $62.40 \pm 1.43$ & & 190 & 0.4 \\
\hline & 52856 & 1280 & Yes & $53.50 \pm 1.38$ & & 132 & 0.3 \\
\hline & 52892 & $610 / 235$ & Yes & $9.24 \pm 0.81 / 4.90 \pm 1.06$ & 0.67 & 73 & $0.7 / 1.30$ \\
\hline & 53037 & 1280 & Yes & $21.14 \pm 0.14$ & & 51 & 0.3 \\
\hline & 53105 & $610 / 235$ & Yes & $19.14 \pm 0.12 / 16.25 \pm 2.23$ & 0.17 & 45 & $1.1 / 1.78$ \\
\hline & 53107 & $610 / 235$ & Yes & $17.16 \pm 0.08 / 15.69 \pm 3.31$ & 0.09 & 70 & $0.8 / 1.21$ \\
\hline & 53127 & $610 / 235$ & Yes & $53.85 \pm 1.26 / 23.51 \pm 3.96$ & 0.87 & 50 & $1.1 / 1.76$ \\
\hline & 53128 & $610 / 235$ & Yes & $38.94 \pm 2.88 / 20.18 \pm 3.22$ & 0.69 & 73 & $0.8 / 1.13$ \\
\hline & 53378 & $610 / 235$ & Yes & $41.45 \pm 1.93 / 14.65 \pm 7.61$ & 1.09 & 130 & $0.9 / 1.36$ \\
\hline & 53379 & $610 / 235$ & Yes & $39.34 \pm 0.72 / 13.32 \pm 5.06$ & 1.13 & 130 & $0.7 / 1.27$ \\
\hline & 53392 & $610 / 235$ & Yes & $36.04 \pm 1.83 / 11.18 \pm 3.83$ & 1.23 & 170 & $0.9 / 1.26$ \\
\hline & 53393 & $610 / 235$ & Yes & $53.84 \pm 3.04 / 49.24 \pm 9.63$ & 0.09 & 170 & $0.8 / 1.33$ \\
\hline \multirow[t]{9}{*}{ Sco X-1 } & 52796 & 1280 & Yes & 38.48 & & & 0.21 \\
\hline & 52798 & 1280 & Yes & 45.52 & & & 0.09 \\
\hline & 52799 & 1280 & Yes & 58.51 & & & 0.16 \\
\hline & 52849 & 1280 & Yes & 44.94 & & & 0.34 \\
\hline & 52855 & 1280 & Yes & 8.04 & & & 0.08 \\
\hline & 52897 & $610 / 235$ & Yes & $6.48 / \leq 5.64$ & & & $0.85 / 1.88$ \\
\hline & 53377 & $610 / 235$ & Yes & $24.75 / \leq 4.26$ & & & $1.06 / 1.42$ \\
\hline & 53391 & $610 / 235$ & Yes & $41.25 / \leq 6.27$ & & & $1.08 / 2.09$ \\
\hline & 53392 & $610 / 235$ & Yes & $51.61 / \leq 3.69$ & & & $0.91 / 1.23$ \\
\hline \multirow[t]{14}{*}{ SS433 } & 52459 & 1280 & Yes & $695.60 \pm 3.40$ & & & 0.45 \\
\hline & 52479 & $610 / 235$ & Yes & $1473.64 \pm 2.04 / 2972.50 \pm 1.20$ & -1.05 & & $0.85 / 1.78$ \\
\hline & 52694 & $610 / 235$ & Yes & $1769.68 \pm 1.70 / 3830.60 \pm 3.43$ & -0.81 & & $1.26 / 1.73$ \\
\hline & 52699 & $610 / 235$ & Yes & $985.53 \pm 6.90 / 2282.50 \pm 7.41$ & -0.88 & & $1.05 / 1.89$ \\
\hline & 52702 & $610 / 235$ & Yes & $1137.15 \pm 6.47 / 2740.19 \pm 9.10$ & -0.92 & & $1.13 / 2.20$ \\
\hline & 52707 & $610 / 235$ & Yes & $1284.78 \pm 3.40 / 2715.50 \pm 9.02$ & -0.78 & & $0.86 / 2.18$ \\
\hline & 52849 & 1280 & Yes & $632.02 \pm 1.27$ & & & 0.20 \\
\hline & 52855 & 1280 & Yes & $660.00 \pm 0.14$ & & & 0.24 \\
\hline & 52897 & $610 / 235$ & Yes & $891.68 \pm 4.08 / 2152.50 \pm 5.43$ & -0.92 & & $0.71 / 1.66$ \\
\hline & 53102 & $610 / 235$ & Yes & $1467.10 \pm 11.47 / 2982.55 \pm 11.01$ & -0.74 & & $0.91 / 1.75$ \\
\hline & 53104 & $610 / 235$ & Yes & $1587.35 \pm 12.27 / 3080.30 \pm 15.5$ & -0.69 & & $1.02 / 1.66$ \\
\hline & 53107 & $610 / 235$ & Yes & $731.50 \pm 0.14 / 1725.71 \pm 6.43$ & -0.89 & & $0.98 / 2.08$ \\
\hline & 53377 & $610 / 235$ & Yes & $1167.75 \pm 1.36 / 2380.30 \pm 10.1$ & -0.75 & & $1.04 / 2.03$ \\
\hline & 53392 & $610 / 235$ & Yes & $1234.97 \pm 2.78 / 2957.50 \pm 07.3$ & -0.92 & & $1.03 / 1.34$ \\
\hline \multirow[t]{2}{*}{ LS 5039} & 53220 & $610 / 235$ & Yes & $28.55 \pm 3.56 / 65.04 \pm 7.71$ & -0.86 & & $0.92 / 2.06$ \\
\hline & 53377 & $610 / 235$ & Yes & $34.29 \pm 2.34 / 74.56 \pm 5.63$ & -0.81 & & $0.74 / 2.03$ \\
\hline \multirow[t]{2}{*}{ LSI+61303 } & 52891 & $610 / 235$ & Yes & $21.70 \pm 2.21 / 6.27 \pm 2.43$ & 1.30 & & $0.83 / 1.23$ \\
\hline & 53377 & $610 / 235$ & Yes & $87.32 \pm 1.04 / 25.63 \pm 5.44$ & 1.28 & & $0.93 / 1.54$ \\
\hline
\end{tabular}

X-ray light curve with rare flaring events, and (C)- transient $\mathrm{X}$-ray light curve with frequent flaring events, as shown in Fig. 5. The nature of the X-ray light curve of all microquasars are shown in Table 4.

It can be seen from the table that the X-ray light curves in the case of HMXBs are mainly type (A) or (C), except for V4641 Sgr with type (B). There are in all 6 HMXBs that are REXBs as shown in Table 4. Depending upon the nature of radio emission these sources are further classified as: persistent radio and transient radio HMXBs. Five HMXBs, LSI+61303, LS5039, SS433, Cyg X-1, and Cyg X-3 are persistent in radio at high frequencies; i.e. above $2 \mathrm{GHz}, 4$ are persistent in radio at low frequencies below $2 \mathrm{GHz}$, the exception being Cyg X-1 (Pandey et al. 2006b). The transient radio HMXBs show occasional radio detection, only during radio outbursts, as seen in the case of V4641 Sgr and discussed further in detail in Sect. 3.2. 
Table 4. Nature of micro-quasars based on light curve.

\begin{tabular}{llllll}
\hline \hline Source & $\begin{array}{l}\text { Dist. } \\
\text { kpc }\end{array}$ & $\begin{array}{l}\text { Accr. } \\
\text { disc } \\
\text { type }\end{array}$ & $\begin{array}{l}\text { X-ray } \\
\text { type }\end{array}$ & $\begin{array}{l}\text { Radio type } \\
\geq 2 \mathrm{GHz}\end{array}$ & $\begin{array}{l}\text { Radio type } \\
\leq 2 \mathrm{GHz} \\
\mathrm{GMRT}\end{array}$ \\
\hline HMXB & $2 \pm$ & $\mathrm{A}$ & - & $\mathrm{p}$ & $\mathrm{p}$ \\
LSI+61303 & 9.6 & $\mathrm{~B}$ & - & $\mathrm{t}$ & $\mathrm{t}$ \\
V4641 Sgr & 5 & $\mathrm{~A}$ & - & $\mathrm{p}$ & $\mathrm{p}$ \\
LS 5039 & $11 \pm 5$ & $\mathrm{~A}$ & $\mathrm{t}$ & $\mathrm{p}$ & $\mathrm{p}$ \\
SS433 & 10.1 & $\mathrm{C}$ & - & $\mathrm{p}$ & $\mathrm{t}$ \\
Cyg X-1 & 17 & $\mathrm{C}$ & $\mathrm{t}$ & $\mathrm{p}$ & $\mathrm{p}$ \\
Cyg X-3 & & & & & \\
\hline LMXB & $6.9 \pm 0.9$ & $\mathrm{~B}$ & - & $\mathrm{t}$ & $\mathrm{ND}$ \\
XTE J1118+480 & $1.1 \pm 1.4$ & $\mathrm{~A}$ & $\mathrm{p}$ & $\mathrm{p}$ & $\mathrm{NO}$ \\
Cir X-1 & 9.4 & $\mathrm{~B}$ & - & $\mathrm{t}$ & $\mathrm{NO}$ \\
XTE J1550-564 & 1.4 & $\mathrm{~A}$ & - & $\mathrm{p}$ & $\mathrm{p}$ \\
Sco X-1 & 1.42 & $\mathrm{~B}$ & $\mathrm{t}$ & $\mathrm{t}$ & $\mathrm{ND}$ \\
GRO J1655-40 & $7.02 \pm 0.5$ & $\mathrm{~B}$ & - & $\mathrm{t}$ & $\mathrm{t}$ \\
GX339-04 & $5.8 \pm 0.4$ & $\mathrm{~B}$ & $\mathrm{p}$ & $\mathrm{p}$ & $\mathrm{ND}$ \\
1E1740.7-2942 & 8.4 & $\mathrm{~B}$ & $\mathrm{t}$ & $\mathrm{t}$ & $\mathrm{ND}$ \\
XTE J1748-288 & $\geq 4.5$ & $\mathrm{~B}$ & $\mathrm{p}$ & $\mathrm{ND}$ \\
GRS 1758-258 & 8.4 & $\mathrm{~B}$ & $\mathrm{p}$ & $\mathrm{p}$ & $\mathrm{t}$ \\
GRS 1915+105 & $14 \pm 4$ & $\mathrm{C}$ & $\mathrm{t}$ & $\mathrm{t}$ & \\
\hline
\end{tabular}

Ref.: (1)- J. M. Paredes et al. (2005), [arXiv: astro-ph/0409226], Chinese journal of Astron. Astrophys., ND $=$ Not detected, NO = Not observed, $\mathrm{p}=$ persistent, $\mathrm{t}=$ transient.
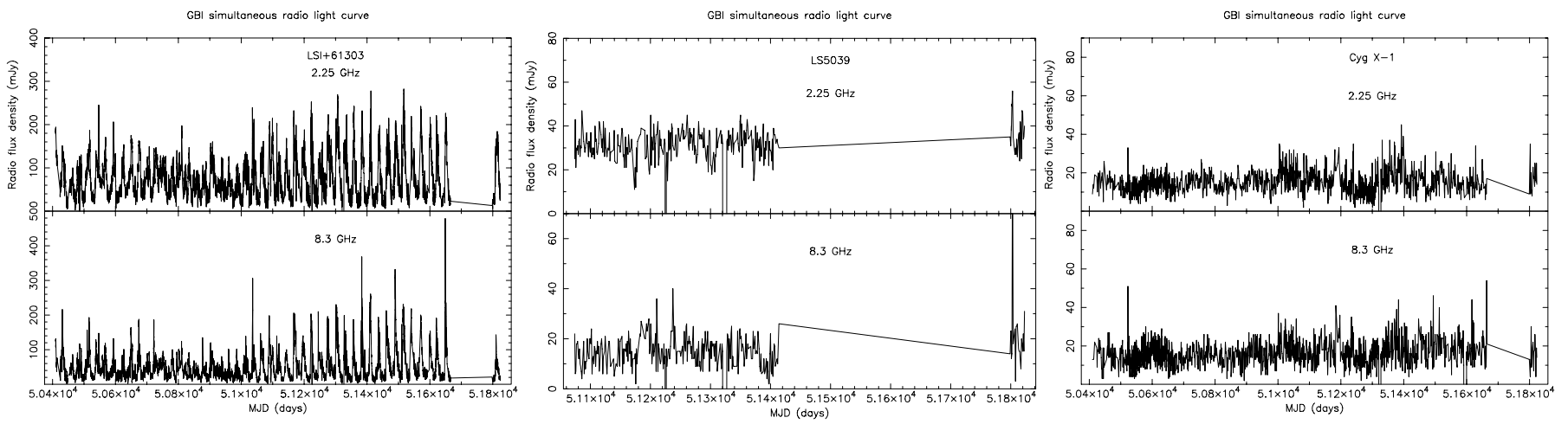

Fig. 2. GBI radio light-curve at 2.2 and $8.2 \mathrm{GHz}$ for persistent radio sources LSI+16303 (left), LS 5039 (middle), and Cyg X-1 (right) showing rare flaring events and detected with GMRT.
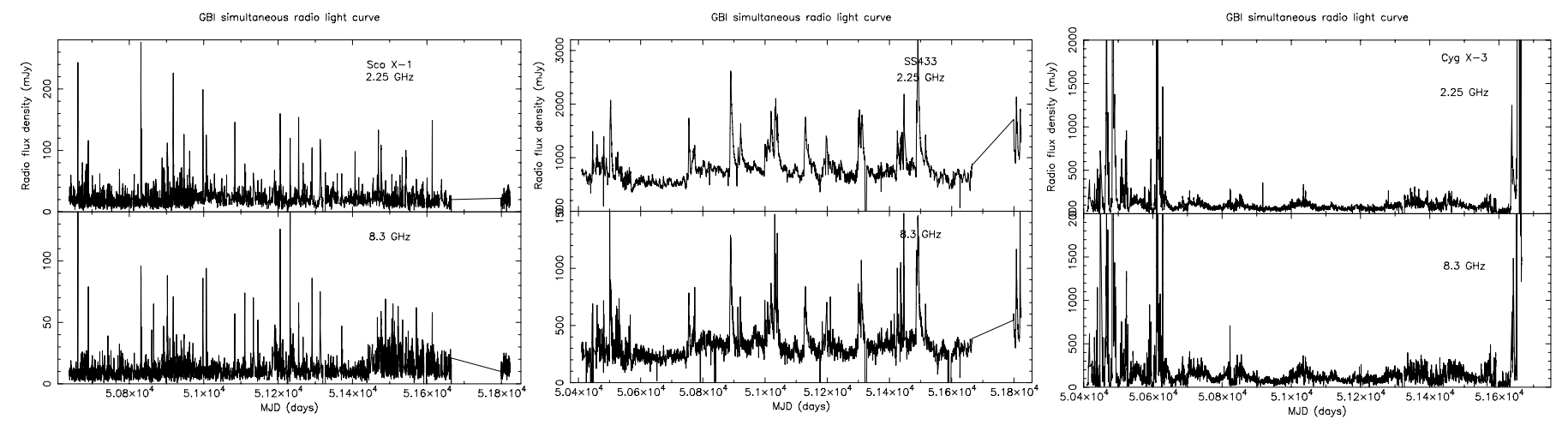

Fig. 3. GBI radio light-curve at 2.2 and $8.2 \mathrm{GHz}$ for persistent radio sources Sco X-1 (left), SS 433 (middle), and Cyg X-3 (right) showing frequent flaring events and detected with GMRT.

In the case of LMXBs, the X-ray light curves are either type (B) or (C), except for Cir X-1 and Sco X-1 with type (A) as seen from Table 4. There are in all 10 LMXBs belonging to this category. They are further grouped into persistent and transient radio LMXBs. The LMXBs, Cir X-1, Sco X-1, XTE J1748-288, and 1E1740.7-2942 are persistent at high radio frequencies, whereas at low radio frequencies only Sco X-1 is persistent (see Sect. 3.2). The microquasars XTE J1748-288 and 1E1740.7-2942 were never detected at GMRT frequencies, and Cir X-1 was, observed with the GMRT due to position constraints. The transient radio LMXBs XTE J1118+480, XTE J1550-564, GRO J1655-40, GX339-04, XTE J1748-288, 

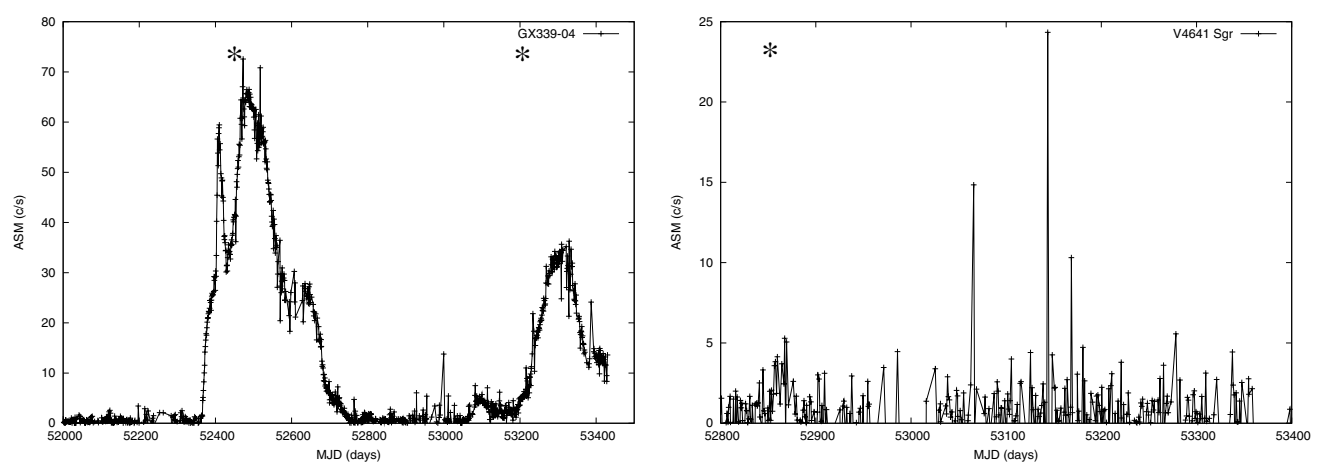

Fig. 4. RXTE/ASM X-ray lightcurve for GX339-04 (left) and V4641 Sgr (right) with positive radio detections marked with $(*)$.
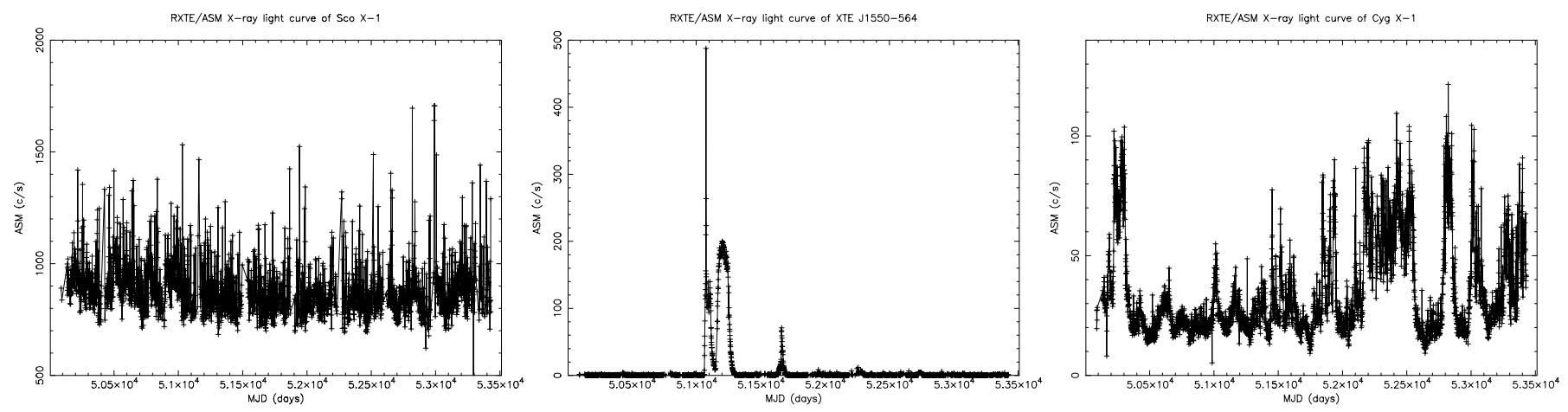

Fig. 5. Persistent (left), quasi-persistent (middle), and transient (right) RXTE/ASM X-ray light-curve of microquasars Sco X-1, XTE J1550-564, and Cyg $\mathrm{X}-1$, respectively.

and GRS 1915+105- were occasionally detected at high radio frequencies, as compared to low frequencies, where only GX339-04 and GRS 1915+105 were positively detected during radio flares. The microquasar XTE J1550-564 was not observed due to position constraints with respect to GMRT. Three microquasars, GRO J1655-40, XTE J1118+480, and XTE J1748-288, were never detected at low frequencies, either due to the SSA effect or due to very faint flux densities at these frequencies.

We applied this correlation to the newly detected possible microquasar candidates IGR J17303-0601 and IGR J184060539, discovered by the INTEGRAL satellite. IGR J17303-0601 is a LMXB that showed transient radio behavior, whereas IGR J18406-0539 is a HMXB that showed persistent behavior in radio regime (Pandey et al. 2006c,d).

A significant variation is seen in the radio flux density of microquasars at low frequencies (see Tables 1-3). This can be a result of the perturbation of the wavefronts from the radio point source by refractive index fluctuations in the turbulent, ionized interstellar medium of our Galaxy. The low-frequency radio data is known to be affected by refractive interstellar scintillation at meter-wavelengths.

\section{Radio properties}

This section discusses about the effect of scintillation on the variations in the flux densities of the microquasars, at very low frequencies.

\subsection{Scintillation effects at low radio frequencies}

There are several classes of flux density variation seen at radio wavelengths. The large-amplitude, broad-band, pulse-to-pulse variations seen for most point sources are thought to be intrinsic to the source emission mechanism. When averaged over many pulses to smooth out these variations, the intensity fluctuations occurring over time scales ranging from minutes to hours, are explained in terms of propagation of radio waves through the irregular interstellar plasma (Scheuer 1968; Rickett 1969). Random variations of electron densities in the interstellar medium (ISM) give rise to phase perturbations, leading to scattering of radio waves. As they propagate, the scattered waves interfere with each other, causing large variations of amplitude with frequency and position. The relative motion between the point source, observer, and the density irregularities translates the spatial amplitude variations into temporal variations at a given point, leading to a typical time scale of intensity fading, called the scintillation time scale. Another observable consequence of this phenomenon is the angular broadening of compact radio sources at low frequencies. Thus the phenomenon of narrow-band intensity fluctuations, i.e. $\sim 100 \mathrm{kHz}$ to a few $\mathrm{MHz}$ and a $100 \%$ modulation, is known as diffractive interstellar scintillation (DISS) (Rickett 1977).

The discovery of long-term ( days to months) flux variations (Cole et al.; Huguenin et al. 1973) and the subsequent correlation of these time scales with the dispersion measure (DM, Sieber 1982) led to the recognition of a second class of propagation effects (Rickett et al. 1984), which has become known as refractive interstellar scintillation (RISS). In RISS, flux variations arise because of focusing and defocusing of the scattered radiation by electron-density irregularities that are large compared to the Fresnel scale. These modulations are fairly broadband in nature. RISS is the preferred explanation of slow flux variability at meter wavelengths (Rickett et al. 1986; Cordes et al. 2004). Besides flux modulations, RISS is also thought to produce slow modulations of decorrelation bandwidth and scintillation time scales.

We carefully investigated the low-frequency GMRT data for microquasars to look for the signatures of radio flux 
Table 5. Scintillation time scale expected at GMRT frequencies.

\begin{tabular}{|c|c|c|c|c|c|c|c|}
\hline Source & $\begin{array}{l}\theta_{0.235} \mathrm{GHz} \\
\text { (mas) }\end{array}$ & $\begin{array}{l}\text { Scintillation- } \\
\theta_{0.61 \mathrm{GHz}} \\
(\mathrm{mas})\end{array}$ & $\begin{array}{l}\text { Angle } \\
\theta_{1.28 \mathrm{GHz}} \\
\text { (mas) }\end{array}$ & $\begin{array}{l}T_{0.235 \mathrm{GHz}} \\
\text { (days) }\end{array}$ & $\begin{array}{l}\text { Scintillation- } \\
T_{0.61 \mathrm{GHz}} \\
\text { (days) }\end{array}$ & $\begin{array}{l}\text { Time } \\
T_{1.28 \mathrm{GHz}} \\
\text { (days) }\end{array}$ & $\begin{array}{l}\text { Variability } \\
\text { (radio) } \\
\text { type }\end{array}$ \\
\hline & HMXB & & & & & & \\
\hline LSI+61303 & 5.46 & 0.67 & 0.13 & 125.83 & 15.44 & 2.99 & $V_{\mathrm{I}} / V_{\mathrm{S}}$ \\
\hline V4641 Sgr & 135.49 & 16.62 & 3.25 & 15612.15 & 1915.08 & 374.49 & $V_{\mathrm{I}}$ \\
\hline LS 5039 & 15.11 & 1.85 & 0.36 & 452.68 & 55.42 & 10.78 & $V_{\mathrm{I}} / V_{\mathrm{S}}$ \\
\hline SS433 & 5.70 & 0.70 & 0.14 & 315.26 & 38.72 & 7.74 & $V_{\mathrm{I}} / V_{\mathrm{S}}$ \\
\hline Cyg X-1 & 2.47 & 0.30 & 0.06 & 56.92 & 6.91 & 1.38 & $V_{\mathrm{I}} / V_{\mathrm{S}}$ \\
\hline Cyg X-3 & 6640 & 814.06 & 159.41 & 765109.33 & 93801.94 & 18368.39 & $V_{\mathrm{I}}$ \\
\hline & LMXB & & & & & & \\
\hline XTE J1118+480 & 9.46 & 1.16 & 0.23 & 218.01 & 26.73 & 6.94 & $V_{\mathrm{I}} / V_{\mathrm{S}}$ \\
\hline Cir X-1 & 144.78 & 17.76 & 3.48 & 9175.43 & 1125.54 & 220.55 & $V_{\mathrm{I}}$ \\
\hline XTE J1550-564 & 132.88 & 16.30 & 3.19 & 8115.04 & 995.45 & 194.81 & $V_{\mathrm{I}}$ \\
\hline Sco X-1 & 12.18 & 1.49 & 0.29 & 392.97 & 48.07 & 9.36 & $V_{\mathrm{I}} / V_{\mathrm{S}}$ \\
\hline GROJ1655 & 46.16 & 5.66 & 1.11 & 1702.05 & 208.69 & 40.93 & $V_{\mathrm{I}}$ \\
\hline GX339-04 & 78.40 & 9.62 & 1.88 & 3613.53 & 443.39 & 86.65 & $V_{\mathrm{I}}$ \\
\hline 1E1740.7-2942 & 450.43 & 55.24 & 10.82 & 44116.56 & 5410.38 & 1059.75 & $V_{\mathrm{I}}$ \\
\hline XTE J1748-288 & 541.39 & 66.39 & 13.00 & 56144.62 & 6884.95 & 1348.16 & $V_{\mathrm{I}}$ \\
\hline GRS $1758-258$ & 330.93 & 40.58 & 7.95 & 32412.34 & 3974.54 & 778.65 & $V_{\mathrm{I}}$ \\
\hline GRS $1915+105$ & 169.94 & 20.84 & 4.08 & 24477.16 & 3001.67 & 587.66 & $V_{\mathrm{I}}$ \\
\hline
\end{tabular}

Ref.: http://rsd-www.nrl.navy.mil/7213/lazio/ne.model/

Table 6. Physical parameters of micro-quasars at GMRT frequencies.

\begin{tabular}{|c|c|c|c|c|c|c|}
\hline Source & $\begin{array}{l}S \\
(\mathrm{mJy})\end{array}$ & $\begin{array}{l}\text { Beam } \\
(\operatorname{arcsec})^{2}\end{array}$ & $\begin{array}{l}v \\
(\mathrm{GHz})\end{array}$ & $\begin{array}{l}T_{\mathrm{B}} \\
(\mathrm{K})\end{array}$ & $\begin{array}{l}B \\
\text { (mGauss) }\end{array}$ & $\begin{array}{l}L_{\text {radio-jet }} \\
\times 10^{29}\left(\mathrm{ergs} \mathrm{s}^{-1}\right)\end{array}$ \\
\hline & HMXB & & & & & \\
\hline LSI+61303 & $87.32 / 25.63$ & $39.12 / 236.47$ & $0.61 / 0.235$ & $\geq 1.86 \times 10^{8} / \geq 1.00 \times 10^{7}$ & $\geq 1.18 / \geq 0.18$ & $2.41 / 0.71$ \\
\hline V4641 Sgr & 49.41 & 139.86 & 1.28 & $\geq 1.86 \times 10^{6}$ & $\geq 0.26$ & 71.5 \\
\hline LS $5039^{\circ}$ & $28.55 / 65.04$ & $58.24 / 159.02$ & $0.61 / 0.235$ & $\geq 2.74 \times 10^{7} / \geq 5.65 \times 10^{7}$ & $\geq 0.55 / \geq 0.22$ & $1.66 / 1.45$ \\
\hline SS433 & $1473.64 / 2972.50$ & $23.94 / 181.31$ & $0.61 / 0.235$ & $\geq 8.38 \times 10^{9} / \geq 1.98 \times 10^{9}$ & $\geq 3.15 / \geq 0.52$ & $234.24 / 182.02$ \\
\hline Cyg X-1 & $9.07 / 11.97$ & $40.68 / 218.84$ & $0.61 / 0.235$ & $\geq 1.78 \times 10^{7} / \geq 5.49 \times 10^{6}$ & $\geq 0.59 / \geq 0.12$ & $0.25 / 1.27$ \\
\hline Cyg X-3 & $41.45 / 14.65$ & $41.93 / 216.19$ & $0.61 / 0.235$ & $\geq 7.68 \times 10^{7} / \geq 6.88 \times 10^{6}$ & $\geq 0.57 / \geq 0.08$ & $28.60 / 3.89$ \\
\hline & LMXB & & & & & \\
\hline XTE J1118+480 & - & - & & - & - & \\
\hline Cir X-1 & - & - & & - & - & 2.99 \\
\hline XTE J1550-564 & - & - & & - & - & \\
\hline Sco X-1 & 41.25 & 50.82 & 0.61 & $\geq 5.20 \times 10^{7}$ & $\geq 0.69$ & 2.23 \\
\hline GRO J1655-40 & - & - & & - & - & \\
\hline GX339-04 & 15 & 16.54 & 1.28 & $\geq 4.06 \times 10^{7}$ & $\geq 1.33$ & 1.52 \\
\hline 1E1740.7-2942 & - & - & & - & - & \\
\hline XTE J1748-288 & - & - & & - & - & \\
\hline GRS $1758-258$ & - & - & & - & - & \\
\hline GRS $1915+105$ & $49.98 / 118.99$ & $50.46 / 145.56$ & $0.61 / 0.235$ & $\begin{array}{l}\geq 6.39 \times 10^{7} / \geq 1.23 \times 10^{8} \\
0.19\end{array}$ & $\geq 0.48 / \geq 0.19$ & $53.9 / 49.4$ \\
\hline
\end{tabular}

density fluctuations due to refractive interstellar scintillation. We estimated the scattering angle and the scintillation time scale for all the microquasars using NE2001 code (http://rsd-www .nrl.navy.mil/7213/lazio/ne.model/) developed to study the interstellar scattering and scintillation of radio wavefronts. Table 5 lists the angular broadening and scintillation time scale calculated for all the microquasars at $0.235,0.61$, and $1.28 \mathrm{GHz}$. It is evident from the table that angular broadening is dominant at lower frequencies. It is also important to note from the table that the LMXBs are more affected by scintillation than HMXBs. This is consistent with the fact that LMXBs are mainly crowded towards the Galactic center, where the electron density irregularities are at their maximum.

At $0.61 \mathrm{GHz}$ the scintillation timescale for Cyg X-1 is at its smallest, so we can confirm from our calculations and series of observations that the flux density fluctuations in the case of Cyg $\mathrm{X}-1$ data are partly affected by isotropic refractive interstellar scintillation (Pandey et al. 2006b).

A similar agreement in the data of SS433 is also noticed at 0.61 and $1.28 \mathrm{GHz}$. In the case of Cyg X-3, the scintillation time scale is at a maximum due to anisotropic interstellar refractive scintillation. The flux density variations seen in the case of Cyg $\mathrm{X}-3$ is intrinsic to the source and independent of interstellar scintillation. This anisotropy is due to the position of the source in the direction of the Cyg OB2 association (Pandey et al. 2006b; Wilkinson et al. 1994, Mioduszewski et al. 2001). In the case of LSI+61303, LS5039, Sco X-1, and XTE J1118+480, the scintillation timescale is small but the flux density variations have not been checked at low frequencies due to non availability of adequate data. However, the flux density variations of about one week at $1 \mathrm{GHz}$, for these sources at their respective distances, 
Table 7. Comparative radio parameters at high frequencies.

\begin{tabular}{lllllll}
\hline \hline Source & $\begin{array}{l}S_{v} \\
(\mathrm{mJy})\end{array}$ & $\begin{array}{l}\text { Size } \\
(\operatorname{arcsec})^{2}\end{array}$ & $\begin{array}{l}v \\
(\mathrm{GHz})\end{array}$ & $\begin{array}{l}T_{\mathrm{B}} \\
(\mathrm{K})\end{array}$ & $\begin{array}{l}L_{\text {radio-jet }} \\
\left(\mathrm{erg} \mathrm{s}^{-1}\right)\end{array}$ & $\begin{array}{l}B \\
(\text { MGAUSS })\end{array}$ \\
\hline LSI+61303 & HMXB & & & & & \\
V4641 Sgr & 41.6 & 0.61 & 5 & $1.85 \times 10^{9}$ & $4.88 \times 10^{29}$ & 51.22 \\
LS 5039 & 16 & 0.250 & 4.9 & $8.35 \times 10^{10}$ & $2.22 \times 10^{32}$ & 159.13 \\
SS433 & 90 & 0.012 & 5 & $6.96 \times 10^{10}$ & $7.61 \times 10^{29}$ & 1226.40 \\
Cyg X-1 & 15 & 0.0005 & 5 & $4.69 \times 10^{11}$ & $1.17 \times 10^{31}$ & 2032.45 \\
Cyg X-3 & 45 & 0.145 & 15 & $1.74 \times 10^{11}$ & $1.59 \times 10^{30}$ & 26203.70 \\
Cyg X-3 & 4000 & 0.016 & 5 & $1.89 \times 10^{9}$ & $7.63 \times 10^{31}$ & 187.07 \\
\hline & LMXB & & & & & \\
XTE J1118+480 & 5.3 & 0.015 & 8 & $7.39 \times 10^{9}$ & $1.73 \times 10^{29}$ & 953.18 \\
Cir X-1 & 280 & 0.2 & 5 & $7.31 \times 10^{10}$ & $4.78 \times 10^{31}$ & 207.40 \\
XTE J1550-564 & 10 & 2 & 8.4 & $1.05 \times 10^{8}$ & $2.66 \times 10^{30}$ & 13.03 \\
Sco X-1 & 8 & 0.0001 & 5 & $4.17 \times 10^{12}$ & $3.55 \times 10^{29}$ & 61552.47 \\
GRO J1655-40 & 2000 & 1 & 1.4 & $1.16 \times 10^{12}$ & $3.24 \times 10^{31}$ & 74.31 \\
GX339-04 & 0.9 & 0.0081 & 8.6 & $2.33 \times 10^{9}$ & $1.40 \times 10^{29}$ & 803.84 \\
GRS 1915+105 & 655 & 1.2 & 8.6 & $1.14 \times 10^{10}$ & $9.95 \times 10^{32}$ & 52.6 \\
\hline
\end{tabular}

Ref.: (1)- J. M. Paredes et al. (2005), [arXiv . astro-ph/0409226], Chinese journal of Astron. Astrophys.

can be due to refractive interstellar scintillation. In the case of all the other microquasars the scintillation timescale is large, meaning that any variability in the flux density seen at low frequencies is due to an intrinsic emission mechanism. In Table 5 the variability due to scintillation is marked as $V_{\mathrm{S}}$ and intrinsic variability of the source is marked as $V_{\mathrm{I}}$.

\subsection{Comparative radio parameters at various frequencies}

It is important to look into the discrepancies of other parameters of microquasars inorder to constrain the emission geometry of these systems.

Tables 6 and 7 list the observed and estimated parameters of all the sources at centimeterand meter-wavelengths. The information in Table 7 has been compiled from the literature. The compilation is neither exhaustive nor complete, but rather representative. The mean radio flux densities quoted in the tables occur during the quiescence or flaring phase of the respective source. The angular size in the case of Table 6 is the size of the synthesized beam because all the sources remained unresolved and in the case of Table 7 it was taken from various references mentioned in the footnote. The following important parameters were calculated using this information:

Brightness temperature, $T_{\mathrm{B}}=\frac{S \lambda^{2}}{2 K \theta^{2}}$

Radio luminosity, $L_{\text {radio-jet }}=4 \pi d^{2} S v$

Equipartition magnetic field, $B=2.3\left(\frac{a A L_{\text {radio-jet }}}{V}\right)^{2 / 7}$

where $\theta$ is the source size, $d$ the distance of the source, and $S$ the flux density. In Eq. (3), a is the ratio of total particle energy to the total electron energy within the emitting source. A constant value of 50 is assumed for this parameter near the Earth (Moffet et al. 1965), $A$ is the constant shape factor of the spectrum $=1.057 \times 10^{12} \mathrm{cgs}$ units (Moffet et al. 1965), and it depends upon the energy and velocity of the emitting particles, and $V$ is the volume of the emitting region. It should be noted that the brightness temperature and equipartition magnetic field mentioned in Table 6 are lower limits since the intrinsic source size is smaller than the synthesized beam used for the calculations.

From Table 6 we note that, for Cyg X-1, Cyg X-3, Sco $\mathrm{X}-1$, and LSI+61303, the radio luminosity decreases as one goes lower in frequencies, i.e., when the radio-emitting plasmon moves from the origin to the base of the jet. However, in a few cases the flux density is rising towards low frequencies, suggesting no absorption effects (e.g.: SS433, LS 5039). Considering this observational result, important information about the magnetic field can be derived. It can be seen that the magnetic field also decreases from the origin to the base of the jet where low frequency radio emissions are generated. The HMXBs have a comparatively higher magnetic field. The source size is inversely proportional to the frequency due to scatter broadening (cf. Tables 6 and 7). As seen in Table 7, the equipartition magnetic field in the case of V4641 Sgr is comparable to LMXBs, and in the case of Sco X-1 is comparable to HMXBs. These calculations suggest that a magnetic field on the order of 10 Gauss and above is necessary for the source to be persistent at radio frequencies. The brightness temperature calculations $\left(\leq 10^{8} \mathrm{~K}\right)$ suggest that the radio emission is nonthermal in origin.

The REXBs with accretion disc type (B) or (C) and with magnetic field on the order of Gauss and below are transient in nature at radio frequencies, cf. Tables 4-7. This information suggests that HMXBs with accretion disc types (A) or (C) are efficient systems and have sufficiently developed magnetic fields to accelerate electrons to higher energies within the jet medium. These populations of electrons will continue to emit at low radio frequencies due to the increased magnetic field even after losing energy due to expansion. In the case of LMXBs, the lowfrequency radio emissions are detected only during flares. Thus important information about the low-frequency emitting region can be derived, which leads to the consideration of various kinds of emission geometry, as seen in the next section.

\section{Radio spectra and the proposed geometry for radio emission at low frequencies}

It is important to follow the several-month spectral evolution of individual sources in the radio window and to construct a general 

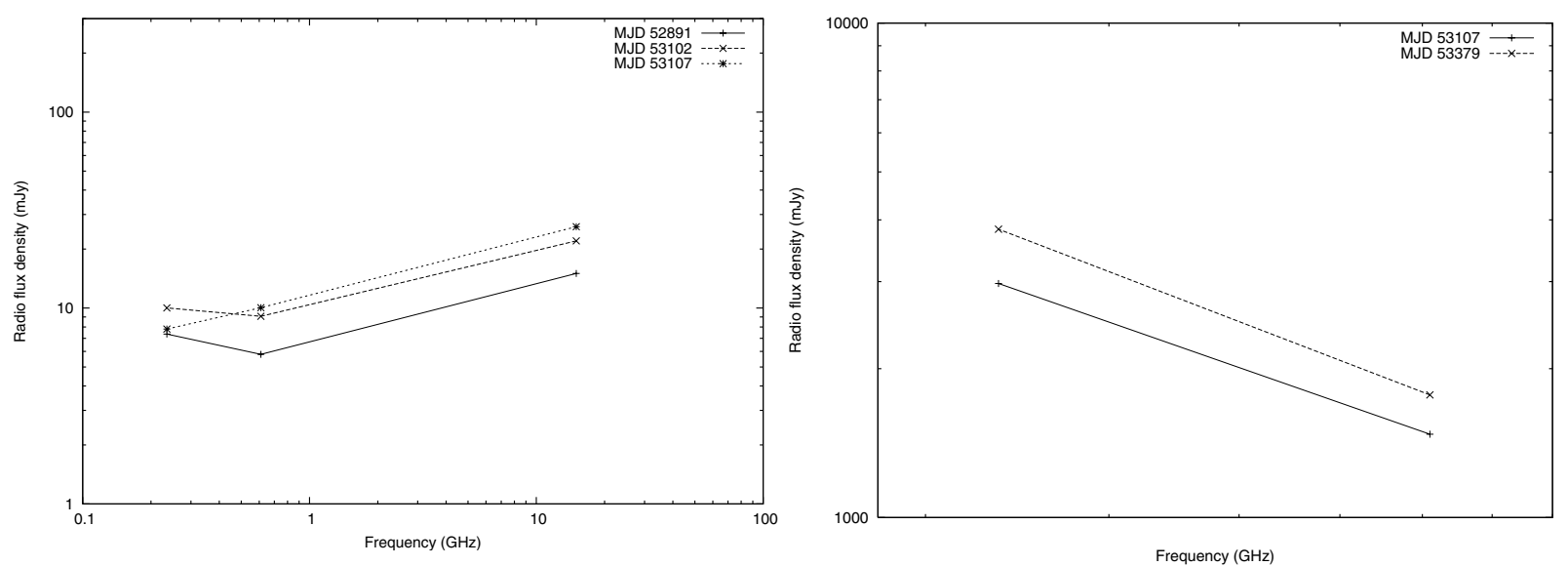

Fig. 6. Radio spectrum of Cyg X-1 (left) and SS433 (right) showing turnover/absorbed and power law decay spectral behavior respectively.

spectral model that can be used to represent the spectra of all the microquasars. The necessary elements of such a model can be deduced from a simple appraisal of the available observational data from Ryle, GBI, and GMRT. A few of the microquasars show low-flux density at lower frequencies as expected from the synchrotron bubble model. This is due to the decrease in the population of energetic electrons as the radio-emitting plasmoid within the jet medium expands from the optically thick to thin medium due to adiabatic expansion. It has long been accepted that radio emission from X-ray binaries is synchrotron emission from material ejected from the system (Hjellming \& Han 1995); Mirabel \& Rodriguez 1994; Fender 2000). However, a simple homogeneous synchrotron-emitting source should exhibit a twocomponent spectrum with a spectral index of +2.5 below some frequency at which self-absorption becomes significant and $\alpha$ above this frequency (with a turnover within one decade in frequency).

We investigated the nature of the radio spectra of microquasars at very low frequencies and have plotted the two typical classes of radio spectra obtained with the GMRT observations. The four sources LSI+61303, Cyg X-1, Cyg X-3, and Sco X-1 show a turnover $\left(S_{v}=v^{\alpha}, \alpha>0\right)$ in the spectrum at low frequencies and two sources; i.e. SS433 and LS5039 show power-law decay spectra $(\alpha<0)$ at low frequencies. The radio spectra of both these classes are shown in the Fig. 6.

The radio spectra of Cyg X-1 and Cyg X-3 have been discussed in detail elsewhere (Pandey et al. 2006b). For LSI+61303 at meter-wavelengths, only 2 observations were performed. As adequate information is not available at low frequencies, the spectral behavior of LSI+61303 will not be discussed in this paper. Based on the radio spectra of microquasars, the emission geometry can be explained by a synchrotron self-absorption model and adiabatic expansion model.

\subsection{Synchrotron self-absorption model}

This is the standard model for considering a compact jet geometry. The higher frequency emission comes from the inner regions of the compact radiating plasma cloud and the lower frequency emission comes from the outer layers due to the increased optical depth for the inner layers (Van der Lann 1966; Hjellming and Johnston 1988). If the expansion of the compact jet is limited by the dense surroundings a synchrotron self absorption will result in a spectral turnover at low radio frequencies. Figure 6 shows a low-frequency turnover in the radio spectra, as expected from a synchrotron bubble model. The spectral index between 0.235 and $0.61 \mathrm{GHz}$ shows a range of $-0.25 \pm 0.15$ for Cyg X-1 whereas the spectral index is $+0.26 \pm 0.10$ between 0.61 and $15 \mathrm{GHz}$. The source has a flat spectral nature at low radio frequencies. Evidently the low-frequency spectrum is partially absorbed due to synchrotron self absorption. It is also possible that the emissions at lower frequencies are dominated by an extended region around Cyg X-1, which may confirm the extended region reported by Gallo et al. (2005), whereas the high frequency emission is dominated by the jet. The microquasars Cyg X-3, LSI+61303, and Sco X-1 show a clear spectral turnover at low frequencies with a spectral index of $\sim 1.8 \pm 0.3$ (cf. Table 3 ). It is interesting to note that in the case of Cyg X-3 a range of variability in the spectral index is seen as the source is highly variable. Thus low frequency observations provide important information about the source and its ambiance.

\subsection{Adiabatic-expansion model}

The next model for radio emissions at low frequencies considers an independent bright emitting region. The radio emission in this case is synchrotron in origin and mainly due to an old population of electrons spiraling in a weak magnetic field and prominently emitting at low frequencies. The radio spectra in this case follow power-law decay, as seen in the case of SS433 and LS 5039 (cf. Fig. 6 left). We also calculated the spectral indices for both the sources from the simultaneous dual-frequency GMRT observations. In the case of SS433, the spectral index between 0.235 and $0.61 \mathrm{GHz}$ ranges from -0.69 to -1.05 , which is likely to be due to marginally different optical- depth conditions at different stages of flaring. In Fig. 6 (left), we have plotted the radio spectra of SS433 during two independent epochs. It can be seen from the figure that the "decay" is the slight steepening from the earlier to the later time, when the radio emitting synchrotron plasmon expands adiabatically and loses its energy at the end of the jet. In the case of LS 5039, the source has a normal steep spectral index of -0.82 . Our result also agrees with the nature of these sources at other wavelengths. The non-thermal radio emission can get Compton-inverse scattered within the dense ISM and give rise to gamma-radiation for LS 5039. Thus, LS 5039 is an interesting source for multi-wavelength studies.

The radio spectra of the transient radio sources that were never detected during GMRT observations, XTE J1118+480, 
XTE J1748-288, GRO J1655-40, 1E1740.7-2942, and GRS $1758-258$, as plotted with the upper limits, also agree with the adiabatic expansion model.

\section{Discussion}

This paper presents the results of the first-ever low- frequency radio observations of the Galactic micro-quasars with the GMRT. The observations were carried out at $0.235,0.610$, and $1.28 \mathrm{GHz}$, and the archival GBI data at 2.3 and $8.2 \mathrm{GHz}$ were considered. We also used the Ryle telescope data at $15 \mathrm{GHz}$ to derive some important information at centimeter wavelengths. A detailed study of the radio spectra of micro-quasars was carried out in this paper. The RXTE/ASM X-ray data were used for this work to derive the X-ray state of the source. The results of our study are listed below.

- The X-ray light-curve reveals that micro-quasars have 3 classes of accretion disc types; (A)- with no flaring events, (B)- with rare flaring events, and (C)- with frequent flaring events. Most of the HMXBs have accretion disc types (A) and (C), and they are persistent at radio frequencies, except for V4641 Sgr with accretion disc type (B), which is transient at radio frequencies. LMXBs have accretion disc types (B) and (C) and are transient in nature at radio frequencies, except for Sco X-1 with accretion disc type (A), which is persistent in nature at radio wavelengths.

- A few micro-quasars, e.g., GRO J1655-40, XTE J1118+480, 1E1740.7-2942, GRS 1758-258, and XTE J1748-288, were never detected by the GMRT. This suggests that either these sources are self-absorbed at GMRT frequencies or are too faint to be detected at the GMRT frequencies. It is also possible that there was no X-ray activity during our radio observations, so these sources were not emitting in radio.

- Persistent micro-quasars like 1E1740.7-2942 and GRS 1758-258, which are weak radio emitters at high radio frequencies, showed no radio detection at meter-wavelengths suggesting that the radio spectra are flat or absorbed. Since, these sources are very faint at high frequencies, it is possible that they are buried in the noise at low radio frequencies.

- GX339-04 and V4641 Sgr were detected on two occasions during radio flares. These flares followed X-ray activity, when the sources went into low/hard X-ray states.

- The persistent radio sources, Cyg X-1, Cyg X-3, LSI+61303, and Sco X-1, emit at meter-wavelengths and show synchrotron self absorption at these frequencies. Sco X-1 is selfabsorbed below $0.61 \mathrm{GHz}$.

- LS 5039 and SS433 show higher flux at $0.235 \mathrm{GHz}$ as compared to $0.61 \mathrm{GHz}$, with a spectral index of $\sim-0.85$. This suggests that there is no turnover in these sources up to $0.235 \mathrm{GHz}$. More low-frequency observations at $150 \mathrm{MHz}$ or lower may help to locate the turnover frequency.

- Two transient radio sources, XTE J1550-564 and Cir X-1, were not observed by GMRT due to declination constraints.

- Significant variability is seen in the low-frequency data on these sources, so we checked for the effects of refractive interstellar scintillation. It was found that Cyg X-1 and SS433 are most likely affected by scintillation. LSI+61303, LS 5039, Sco X-1, and XTE J1118+480 may be affected by scintillation; however, detailed series of observations need to be performed at low frequencies to check this effect. Most of the other micro-quasars appear not to be affected by refractive interstellar scintillation.
- Some of the sources showed spectral index evolution during the flaring activities, strongly suggesting the ejection of expanding blobs of plasma. In these cases, the radio jet is optically thick at low radio frequencies during the initial phases of the radio outbursts and subsequently becomes optically thin as the flare evolves.

- Important radio parameters, such as size, brightness temperature, equipartition magnetic field and radio luminosity, were estimated. The brightness temperature suggests that the radio emission is synchrotron in origin. However, at meter wavelengths only an upper limit to the brightness temperature and equipartition magnetic field was obtained, since the source remained unresolved and the size of the synthesized beam was used. From the VLBI observations at centimeter wavelengths, it is clear that the equipartition magnetic field is high for HMXBs as compared to LMXBs. Also the size of the source increases at low frequencies due to angular broadening.

These monitoring results thus suggest that at low frequencies, a few sources follow the synchrotron spectra and show absorption effects; however, a few sources do not follow the absorption trends and emit at unexpectedly higher flux densities. The sources that do not show absorption effects at low frequencies give the signature of extended regions around the source, e.g, SS433, Cyg X-1, etc. More systematic observations of microquasars at low radio frequencies is very important in various radio states to acquire homogeneous data so that most of the theoretical suggestions can be confirmed. Simultaneous radio data at various frequencies is necessary to obtain reliable life times, size, and various jet parameters of the varying components.

Acknowledgements. M.P. thanks the staff of the GMRT, who made these observations possible. The GMRT is run by the National Centre for Radio Astrophysics of the Tata Institute of Fundamental Research. M.P. is grateful to Director of the NCRA for providing the research facilities in Pune and to Prof. V. H. Kulkarni of Mumbai University for his encouragement. This research is supported by ISRO, grant No. DOS. 9/2/109/2003-II under its RESPOND program. M.P. also acknowledges the support provided by Prof. P. O. Lagage, at the CEA, during her visit and stay in France, and would also like to thank Prof. J. M. Paredes, Dr. S. Corbel, Prof. J. Osborne, and Prof. R. Spencer for their comments on this paper.

\section{References}

Cordes J. M 2004, Milky Way Surveys: The Structure and Evolution of our Galaxy, Proc. The 5th Boston University Astrophysics Conference, ASP Conf. 317,211

Cole, T. W., Hesse, H. K., \& Page, C. G., 1970, Nature, 225, 712

Corbel, S., \& Fender, R. P., 2002, ApJ, 573, L35-L39

Fender, R. P., \& Hendry, M. A. 2000, MNRAS, 317

Gallo, E., Fender, R., Kaiser, C. Russell et al. Nature, 438, 819

Han, Jin-Lin, 1995, ChA\&A, 19, 285

Haardt, F., \& Maraschi, L, 1991, ApJ, 380, L51

Hjellming R., \& Johnston J., 1988, ApJ, 328, 600

Huguenin, G. R., Taylor, J. H., \& Helfand, D. J, 1973, ApJ, 181, L139

Ishwara-Chandra, C. H., Yadav, J. S., \& Pramesh Rao, A. 2002, A\&A, 388, L33

Ishwara-Chandra, C. H., \& Pramesh Rao, A. 2005, ChJAA, 5, 269

Ishwara-Chandra, C. H., Pramesh R., A. Pandey, M., \& Manchanda, R. K., Durouchoux, Ph. 2005, ChJAA, 5S, 87

Markoff, S., Nowak, M., Corbel, S., Fender, R., \& Falcke, H. 2003a, A\&A, 397, 645

Markoff, S., Nowak, M., Corbel, S., Fender, \& Rob, F. 2003b, NewAR, 47, 491 
Mioduszewski, A. J., Rupen, M. P., Hjellming, R. M., et al. 2001, ApJ, 553, 766

Mirabel, I. F., \& Rodriguez, L. F. 1994, Nature, 371, 46

Mirabel, I. F., \& Rodríguez, L. F. 1999, ApJ, 511, 398

Moffet, A. 1962, APJS., 7, 93

Pandey, M. D., Durouchoux, Ph., Manchanda, R. K. et al. Pooley, G. 2004, Inun. Conf, 699

Pandey, M., Rao, A. P., \& Ishwara-Chandra, C. H. 2006a, Low frequency observations of GRS $1915+105$, in preparation

Pandey, M., Rao, A. P., Pooley, G. G., et al. 2006b, A\&A, 447, 525

Pandey, M., Manchanda, R. K., Rao, A. P., Durouchoux, \& P., Ishwara-Chandra 2006c, A\&A, 446, 471

Pandey, M., Rao, A. P., Manchanda, R., Durouchoux, P., \& Ishwara-Chandra, C. H. 2006d, A\&A, 453, 83
Paredes, J. M. 2005, Ap\&SS, 300, 267 [arXiv:astro-ph/0409226] Poutanen, J., \& Svensson, R. 1996, ApJ, 470, 249

Rickett, B. 1969, Nature, 221, 158

Rickett, B. 1977, ARA\&A, 15, 479

Rickett, B. 1984, A\&A, 134, 390

Rickett, B. 1986, ApJ, 307, 564

Scheuer, P. A. G. 1968, ApJ, 151, L139

Shakura, N. I., \& Sunyaev, R. A. 1973, A\&A, 24, 337

Shapiro, S. L., Lightman, A. P., \& Eardley, D. M. 1976, ApJ, 204, 187

Sieber, W. 1982, A\&A, 113, 311

Sunyaev, R. A., \& Titarchuk, L. G. 1980, A\&A, 86, 121

Van der Laan, H. 1966, Nature, 211, 1131

Wilkinson, P. N., Narayan, R., \& Spencer, R. E. 1994, MNRAS, 269, 67 
M. Pandey et al.: Low-frequency radio monitoring of microquasars, Online Material $p 1$

\section{Online Material}


M. Pandey et al.: Low-frequency radio monitoring of microquasars, Online Material $p 2$

Table 2. Details of observations on transient radio source V4641 Sgr and GX339-04 with the GMRT.

\begin{tabular}{|c|c|c|c|c|c|}
\hline Source & MJD & $\begin{array}{l}\text { Freq. } \\
\text { (MHz) }\end{array}$ & Detection & $\begin{array}{l}S_{v} \\
(\mathrm{mJy})\end{array}$ & $\begin{array}{l}\mathrm{rms} \\
\left(\mathrm{mJy}^{-1}\right)\end{array}$ \\
\hline \multirow[t]{9}{*}{ V4641 Sgr } & 52849 & 1280 & No & $<0.6$ & 0.20 \\
\hline & 52855 & 1280 & Yes & $49.41 \pm 1.13$ & 0.40 \\
\hline & 52897 & $610 / 235$ & No & $<2.50 /<3.87$ & $0.83 / 1.29$ \\
\hline & 53107 & $610 / 235$ & No & $<3.36 /<5.34$ & $1.12 / 1.78$ \\
\hline & 53126 & $610 / 235$ & No & $<2.88 /<4.11$ & $0.96 / 1.37$ \\
\hline & 53377 & $610 / 235$ & No & $<2.64 /<4.35$ & $0.88 / 1.45$ \\
\hline & 53379 & $610 / 235$ & No & $<3.27 /<6.27$ & $1.09 / 2.09$ \\
\hline & 53391 & $610 / 235$ & No & $<3.12 /<6.12$ & $1.04 / 2.04$ \\
\hline & 53392 & $610 / 235$ & No & $<2.82 /<3.87$ & $0.94 / 1.29$ \\
\hline \multirow[t]{22}{*}{ GX339-04 } & 52459 & 1280 & Yes & $15.20 \pm 0.30$ & 0.15 \\
\hline & 52694 & $610 / 235$ & No & $<3.15 /<6.09$ & $1.05 / 2.03$ \\
\hline & 52699 & $610 / 235$ & No & $<3.12 /<4.56$ & $1.04 / 1.52$ \\
\hline & 52702 & $610 / 235$ & No & $<3.63 /<5.4$ & $1.21 / 1.8$ \\
\hline & 52707 & $610 / 235$ & No & $<2.4 /<6.48$ & $0.80 / 2.16$ \\
\hline & 52796 & 1280 & No & $<0.24$ & 0.08 \\
\hline & 52799 & 1280 & No & $<0.18$ & 0.09 \\
\hline & 52829 & $610 / 235$ & No & $<3.03 /<3.69$ & $1.01 / 1.23$ \\
\hline & 52849 & 1280 & No & $<0.33$ & 0.11 \\
\hline & 52855 & 1280 & No & $<0.24$ & 0.08 \\
\hline & 52897 & $610 / 235$ & No & $<3.27 /<3.69$ & $1.09 / 1.23$ \\
\hline & 53102 & $610 / 235$ & No & $<2.70 /<3.27$ & $0.90 / 1.09$ \\
\hline & 53104 & $610 / 235$ & No & $<2.61 /<4.02$ & $0.87 / 1.34$ \\
\hline & 53106 & $610 / 235$ & No & $<3.12 /<5.37$ & $1.04 / 1.79$ \\
\hline & 53107 & $610 / 235$ & No & $<2.34 /<3.48$ & $0.78 / 1.16$ \\
\hline & 53126 & $610 / 235$ & No & $<4.20 /<6.27$ & $1.40 / 2.09$ \\
\hline & 53181 & $610 / 235$ & No & $<2.94 /<6.03$ & $0.98 / 2.01$ \\
\hline & 53220 & $610 / 235$ & Yes & $4.20 \pm 0.12 /<6.09$ & $0.96 / 2.03$ \\
\hline & 53377 & $610 / 235$ & No & $<2.82 /<5.10$ & $0.94 / 1.70$ \\
\hline & 53379 & $610 / 235$ & No & $<3.09 /<4.08$ & $1.03 / 1.36$ \\
\hline & 53391 & $610 / 235$ & No & $<3.12 /<6.27$ & $1.04 / 2.09$ \\
\hline & 53392 & $610 / 235$ & No & $<2.49 /<6.06$ & $0.83 / 2.02$ \\
\hline
\end{tabular}

